\title{
Combining Ability Studies in Cotton Interspecific Heterotic Group Hybrids (G. hirsutum x G. barbadense) for Seed Cotton Yield and its Components
}

\author{
S. Rajeev ${ }^{1}$ and S.S. Patil ${ }^{1}$ \\ ${ }^{1}$ Department of Genetic and Plant Breeding, University of Agricultural Sciences, Dharwad, \\ Karnataka, 580005, India \\ *Corresponding author
}

\section{A B S T R A C T}

Interspecific hybrids of hirsutum and barbadense are known for high productivity and fibre quality. Estimation of combining ability of a line or inbred is useful for predicting its useful as parent in hybrid breeding. The analysis of general and specific combining ability

\section{Keywords}

Heterotic group, Interspecific hybrids, Combining ability and Cotton.

Article Info

Accepted:

26 June 2018

Available Online:

10 July 2018 aids in identification of potential parents for production of superior hybrids. General and specific combining ability effects were estimated for eight lines, four testers and their 32 hybrids. The results of the present investigation on combining ability effects revealed that among the hirsutum lines, DHMS is a good general combiner for most of the characters viz., seed cotton yield, lint yield, number of bolls per plant, boll weight, number of sympodia, sympodial length at 50 per cent plant height, ginning outturn (\%) with significant positive gca effects. Another hirsutum line DH2572 exhibited significant gca effects for seed cotton yield and lint yield. Among the barbadense testers, DB534 exhibited significant gca effects for most of the characters viz., seed cotton yield, lint yield, boll weight plant height and number of sympodia while the tester SNICB75-10 exhibited significant gca effects for seed cotton yield, lint yield and plant height. Among the crosses, the cross DHMS x SNICB75-10 and DH2572 x SNICB75-10 recorded significant sca effects for seed cotton yield, lint yield, number of bolls per plant, number of sympodia, sympodial length at 50 per cent plant height and SPAD meter reading confirming the role of non-additive effects in governing inheritance of these quantitative characters in these crosses which can be exploited by hybrid breeding.

\section{Introduction}

India as a developing country is more dependent on agriculture followed by the Textile industry, where it is significantly cotton based, for employment generation to nearly 60 million persons directly or indirectly. It contributes to $14 \%$ of industrial production and $4 \%$ of GDP and majorly $35 \%$ of total exports and accounts for about 16 per cent of India's export earnings (Anonymous, 2017). This indicates that the overall development of the Indian economy is strongly dependent on cotton, the king of the natural fibers as Indian textile industry is predominantly cotton based industry till date due to its inherent eco-friendly and comfort characteristics. 
In the history of Indian cotton, intra hirsutum hybrids lead to a significant impact on increasing cotton production also lead to acute shortage of ELS cotton in the country. Even though India boasts of cotton exports, import of ELS cotton has become inevitable due to drastic reduction in the production of extralong staple and short staple cotton. Since long period genetic improvement of barbadense varietal lines has been very limited. Hence, to frame research priorities on improving potentiality of barbadense varietal base and developing hybrid oriented populations based on them and utilizing them in deriving potential interspecific hybrids.

Allard (1960) indicated that, selection of parents on the basis of per se performance is not rewarding where combining ability of line or inbred is the important factor in determining future usefulness of the lines for developing hybrids. Studies on combining ability are useful to understand the nature of genetic variance. It helps the breeder to choose suitable parents for developing either hybrids or varieties. The concepts of general and specific combining ability were first introduced by Sprague and Tatum (1942), who designated general combining ability (GCA) as the average performance of a line in all the possible hybrid combinations and specific combining ability (SCA) was applied to those cases where certain hybrid combinations did relatively better or worse than would be expected on the basis of the average performance of the lines involved. $\mathrm{L} \times \mathrm{T}$ analysis (Kempthore, 1957) is useful technique suitable for identification of cross combination and parents to be used in crossing programme for hybrid breeding.

\section{Materials and Methods}

The experimental material comprised of eight hirsutum lines viz., DH2752, DHMS, RAH13-86, RAH-16, RAH-25-17, RAH-370,
RAH-5-10 and DH-37 and four barbadense testers viz., DB534, SNICB75-10, RAB-4 and RAB-8 were crossed to generate 32 interspecific heterotic group crosses in line $\mathrm{x}$ tester design. During 2010-11 attempt was made to identify heterotic box based on detailed line $\mathrm{X}$ tester study of interspecific crosses involving members of opposite heterotic groups (Table 1). To reconfirm the potentiality of heterotic box, detailed evaluation of interspecific hybrids involving these lines along with other crosses were taken up during 2013-2014. Based on both studies two barbadense SNICB 75-10 (B1) and DB 534 (B2) and two hirsutum DH2752 (H1) and DHMS (H2) lines giving best hybrids ( $\mathrm{H} \mathrm{x} \mathrm{B}$ ), combination between them were selected based on predicted double cross performance of top elite combiners.

In this experiment, 32 interspecific hybrids (G.hirsutum $x$ G.barbadense) along with two checks (RAHB 87 and DCH 32) subjected to Line $\mathrm{x}$ Tester analysis ( 8 hirsutum lines and 4 barbadense lines), was laid out in Randomized Block Design (RBD) with two replications. Each entry was sown in 2 row plots spaced at $90 \mathrm{~cm}$ with recommended dose of fertilizer and seeds were sown on 21-62014, 2-3 seeds were dibbled per spot in each row and thinning was attended to retain one healthy plant per hill at 25 days after sowing. All the recommended package of practices was followed to rise healthy crop. The mean value of five random plants in $F 1 \mathrm{~s}$, and parental lines were employed for Line $\mathrm{x}$ tester statistical analysis and combining ability estimation in Indo stat package. Observations were recorded on three randomly selected plants of each entry. Observations were recorded on Thirteen different quantitative characters viz., seed cotton yield, lint yield, number of bolls per plant, boll weight, plant height, number of monopodia, number of sympodia, Sympodial length at 50 percent plant height, ginning out turn, SPAD meter 
reading, inter branch distance, lint index and seed index were studied. The performance of crosses with respect to seed cotton yield was considered in as prime trait for assessing combining ability of different possible heterotic boxes.

\section{Results and Discussion}

The analysis of variance (ANOVA) for combining ability for different traits is presented in Table 1. The mean sum of squares due to lines were significant for the most of the characters studied except for reproductive points on sympodia, number of monopodia, number of sympodia and ginning outturn (\%). The mean sum of squares due to line was significant for most of the characters studied except for SPAD meter reading and inter branch distance (\%) and seed index. The mean sum of squares due to tester was significant for most of the characters studied except for ginning per cent, SPAD meter reading and inter branch distance $(\%)$, lint index and seed index. The interaction effect of lines and testers was found to be significant for all the characters except seed index.

The magnitude of SCA variance was greater than GCA variance for all the characters studied. The variance ratios were less than half in all the characters indicating that dominance variance was more than additive variance. A higher proportion of SCA variance for most of the characters indicated that non-additive and additive $\mathrm{x}$ non-additive type of interactions were significantly higher among hybrids where, non-additive gene action can be exploited by heterosis breeding. Similar results were obtained by Kaushik and Kapoor (2007) and Pranesh (2014). The estimates of general combining ability effects of lines and testers are presented in Table 2. While, their specific combining ability effects are presented in Table 3 for all the thirteen characters studied.
Among eight lines, DHMS (156.06) and DH2752 (251.23) exhibited significant gca effects in positive direction showing predominance of additive genes for the trait. However, RAH-5-10 (-408.75) recorded negative significant gca effects. Out of four testers, DB534 (178.84) and SNICB75-10 (46.46) had significant positive while RAB4 (151.75) and RAB8 (-73.54) had significant negative gca effects. Thirty one crosses differed significantly for sca effects, of these sixteen were positive, fifteen were negative of which lowest sca $(-577.5)$ was noticed in RAH-16 $x$ DB534. The top three best combiners that registered maximum positive sca effects were RAH-25-17 x DB534 (688.5), VB-37 x RAB-8 (596.6) and RAH-16 x RAB4 (512.5) indicating the non-additive and additive $\mathrm{x}$ non-additive type of interactions were significantly higher among hybrids, thus non-additive gene action could be exploited by heterosis breeding. These results are in conformity with the findings of Rama Krishna (2008) and Pranseh (2014) for seed cotton yield.

Similarly for lint yield, lines viz., DHMS (240.07) and DH2752 (84.74) exhibited significant gca effects in positive direction and RAH-5-10 (-145.28) recorded negative significant gca effects. Out of four testers, DB534 (46.04) and SNICB75-10 (46.04) had significant positive while RAB4 (-63.34) and RAB8 (-17.09) had significant negative gca effects. Twenty nine crosses differed significantly for sca effects, of these thirteen were positive sixteen were negative of which lowest sca (-577.5) was noticed in VB-37 $x$ SNICB75-10. The top three best combiners that registered maximum positive sca effects were RAH-25-17 x DB534 (213.1), VB-37 x RAB-8 (208.9) and RAH-13-86 x RAB-4 (197.9).

Among the lines, DHMS (9.57) recorded significant gca effect in positive direction for 
number of bolls per plant while RAH-16 (5.67) and RAH -5-10 (-4.14) contributed significant gca effect in negative direction. Among testers, DB534 (3.30) exhibited significant gca effect in positive direction and RAB-4 (-3.97) showed significant negative gca effects. Eight out of seventeen crosses exhibited significant positive sca effects, among them, top three crosses were RAH-13$86 \times$ RAB8 (7.82), DH2752 x SNICB75-10 (6.74) and DHMS $x$ SNICB75-10 (5.61) showed significant sca effect in positive direction in that order. For boll weight, hirsutum line DHMS (1.24) had significant positive gca effects among lines and none of the testers showed significant gca effects. Only the cross RAH-25-17 x DB534 exhibited significant sca effect in positive direction. Similar results were also narrated by Reddy (2001), Neelima (2002), Pole et al. (2008) and Pranesh (2014) for both traits viz., number of bolls per plant and boll weight.

The lines RAH-25-17 (8.56) and RAH-5-10 (7.94) were very good general combiner for plant height. The line RAH-16 (-12.81) and DHMS (-12.31) showed significant negative gca effect. Two testers viz., DSC-31 (7.70) showed significant positive gca effects. Whereas, two testers viz., DB534 (4.06) and SNICB75-10 (3.63) showed significant positive gca effect while the tester RAB-8 (6.25) exhibited significant negative gca effect. Twelve crosses showed significantly positive sca effects ranging from 5.75 (RAH-13-86 x RAB8) to 24.38 (RAH-16 x RAB8). Seven crosses contributed significant sca effects towards negative direction with a range of 31.63 (DHMS $x$ RAB8) to -3.84 (VB-37 $x$ RAB-8).Similar results were reported by Maisuria et al. (2006), (2009), Patil et al. (2009) and Pranesh (2014).

None of the lines or testers had significant gca effects for number of monopodia per plant. Among crosses, sca effects were in the range of -1.27 (DHMS x SNICB75-10) to 0.68 (VB-
37 x SNICB75-10) and only one cross viz., DHMS $x$ SNICB75-10 had significant negative sca effects for this trait. Similar to these results sca effects in negative direction reported by Shanmugavalli and Vijendradas (1995) Reddy (2001), Nidagundi (2010) and Yanal (2013). As monopodia are vegetative branches consuming more photosynthate,less number of monopodia but with more sympodia is desirable for interspecific hybrids.

For number of sympodia per plant hirsutum lines viz., DHMS (4.99) and RAH-13-86 (3.78) contributed significant positive gca effects, whereas, RAH-16 (-4.86) and RAH$370(-2.57)$ exhibited significant negative gca effects. Only one testers, DB534 (2.57) showed significant positive gca effect. Six crosses contributed significant positive sca effects ranging from 3.11 (RAH-370 x DB534) to 5.42 (DHMS $x$ SNICB75-10). Eight crosses showed significantly negative sca effects ranging from -2.45 (DHMS $x$ DB534) to -6.10 (VB-37 $x$ SNICB7510).Similar results were reported by Kajjidoni (1997), Neelima (2002), Maisuria et al., (2006) and Saifullah et al., (2014) for this trait.

Three lines DHMS (4.18), RAH-370 (2.07) and RAH-5-10 (2.03) exhibited significant positive gca effects for sympodial length at 50 per cent plant height Whereas, two lines viz., VB-37 (-7.91) and RAH-16 (-3.37) recorded significant negative gca effects. Among testers only one tester DB534 (-2.01) exhibited significant negative gca effects. Eleven crosses contributed significant positive sca effects ranging from 2.88 (RAH-16 x DB534) to 9.17 (DHMS x SNICB75-10). Twelve crosses showed significantly negative sca effects ranging from -2.57 (RAH-370 x RAB4) to -8.31 (RAH-13-86 $x$ SNICB7510).Similar results were quoted by Mallikarjun (2005), Somashekhar (2006) and Ramakrishna (2008) in their studies with this trait. 


\section{Int.J.Curr.Microbiol.App.Sci (2018) 7(7): 3955-3963}

Table.1 ANOVA for combining ability of interspecific heterotic group crosses involving hirsutum and barbadense groups

\begin{tabular}{|c|c|c|c|c|c|c|c|c|c|c|c|c|c|c|}
\hline $\begin{array}{l}\text { Source of } \\
\text { Variation }\end{array}$ & $\mathrm{DF}$ & $\begin{array}{l}\text { Seed cotton } \\
\text { yield (kg } \\
\text { /ha) }\end{array}$ & $\begin{array}{l}\text { Lint yield } \\
\text { (kg /ha) }\end{array}$ & $\begin{array}{l}\text { Number } \\
\text { of bolls } \\
\text { per plant }\end{array}$ & $\begin{array}{l}\text { Boll } \\
\text { weight } \\
\text { (g) }\end{array}$ & $\begin{array}{l}\text { Plant } \\
\text { height } \\
(\mathrm{cm})\end{array}$ & $\begin{array}{l}\text { Number of } \\
\text { monopodia }\end{array}$ & $\begin{array}{c}\text { Number of } \\
\text { sympodia }\end{array}$ & $\begin{array}{c}\text { Sympodial } \\
\text { length at } 50 \\
\% \text { plant } \\
\text { height }(\mathrm{cm})\end{array}$ & $\begin{array}{c}\text { Ginning } \\
\text { outturn } \\
(\%)\end{array}$ & $\begin{array}{c}\text { SPAD } \\
\text { meter } \\
\text { reading }\end{array}$ & $\begin{array}{c}\text { Inter } \\
\text { branch } \\
\text { distance } \\
(\mathrm{cm})\end{array}$ & $\begin{array}{c}\text { Lint } \\
\text { index } \\
(\mathrm{g})\end{array}$ & $\begin{array}{c}\text { Seed } \\
\text { index } \\
(\mathrm{g})\end{array}$ \\
\hline Blocks & 1 & 323347.7 & 19905.4 & 34.7 & 0.1 & 42.3 & 0.03 & 5.9 & 1.6 & 2.8 & 7.4 & 0.03 & 1.0 & 0.8 \\
\hline Due to Males & 3 & $333742.1 *$ & $40563.9 *$ & $156.8 * *$ & $0.8 * *$ & $377.5 * *$ & $0.3 * *$ & $53.1 * *$ & $32.4 * *$ & 3.6 & 1.6 & 0.9 & 0.9 & 0.7 \\
\hline Due to Female & 7 & $889596.0 * *$ & $111515.6^{* *}$ & $166.4 * *$ & $2.9 * *$ & $621.8 * *$ & $0.2 * *$ & $81.4 * *$ & $124.8 * *$ & $17.1 * *$ & 9.0 & 0.7 & $2.7 * *$ & 1.2 \\
\hline $\begin{array}{l}\text { Male X } \\
\text { Female } \\
\end{array}$ & 21 & $388197.7 *$ & $50578.1^{*}$ & $48.0 * *$ & $0.8 * *$ & $580.3 * *$ & $0.5 * *$ & $23.8 * *$ & $77.1 * *$ & $13.5^{* *}$ & $21.0 *$ & $1.2 *$ & $1.7 *$ & 1.7 \\
\hline Error & 43 & 83592.2 & 12334.6 & 16.9 & 0.1 & 42.3 & 0.1 & 5.1 & 6.6 & 1.7 & 5.2 & 0.3 & 0.4 & 1.7 \\
\hline $\mathbf{s}^{2}$ gca & & 18622.6 & 2121.8 & 9.5 & 0.1 & -6.7 & 0.0 & 3.6 & 0.1 & -0.3 & -1.3 & 0.0 & 0.0 & -0.1 \\
\hline $\mathrm{s}^{2} \mathrm{sca}$ & & 122302.7 & 16121.7 & 15.6 & 0.3 & 269.0 & 0.2 & 9.3 & 35.2 & 5.9 & 7.2 & 0.3 & 0.5 & 0.0 \\
\hline $\mathrm{s}^{2} \mathrm{gca} / \mathrm{s}^{2} \mathrm{sca}$ & & 0.2 & 0.1 & 0.6 & 0.3 & 0.0 & -0.1 & 0.4 & 0.0 & 0.0 & -0.2 & -0.1 & 0.0 & 4.4 \\
\hline
\end{tabular}


Table.2 General combining ability effects of parents representing hirsutum and barbadense groups

\begin{tabular}{|c|c|c|c|c|c|c|c|c|c|c|c|c|c|c|}
\hline Sl No. & Lines/Testers & $\begin{array}{c}\text { Seed } \\
\text { cotton } \\
\text { yield (kg } \\
\text { /ha) }\end{array}$ & $\begin{array}{l}\text { Lint yield } \\
\text { (kg/ha) }\end{array}$ & $\begin{array}{l}\text { Number } \\
\text { of bolls } \\
\text { per plant }\end{array}$ & $\begin{array}{l}\text { Boll } \\
\text { weight } \\
\text { (g) }\end{array}$ & $\begin{array}{l}\text { Plant } \\
\text { height } \\
(\mathrm{cm})\end{array}$ & $\begin{array}{l}\text { Number of } \\
\text { monopodia }\end{array}$ & $\begin{array}{c}\text { Number } \\
\text { of } \\
\text { sympodia }\end{array}$ & $\begin{array}{l}\text { Sympodial } \\
\text { length at } \\
50 \% \text { plant } \\
\text { height } \\
(\mathrm{cm})\end{array}$ & $\begin{array}{l}\text { Ginning } \\
\text { outturn } \\
(\%)\end{array}$ & $\begin{array}{l}\text { Spadmeter } \\
\text { reading }\end{array}$ & $\begin{array}{c}\text { Inter } \\
\text { branch } \\
\text { distance } \\
(\mathrm{cm})\end{array}$ & $\begin{array}{l}\text { Lint } \\
\text { index } \\
(\mathrm{g})\end{array}$ & $\begin{array}{c}\text { Seed } \\
\text { index } \\
(\mathrm{g})\end{array}$ \\
\hline \multicolumn{15}{|c|}{ Hirstum Lines } \\
\hline 1 & DH2752 & $422.40 * *$ & $84.74 * *$ & 0.64 & 0.55 & -2.19 & -0.21 & 0.16 & -1.25 & -0.79 & -0.45 & 0.26 & -0.55 & -0.58 \\
\hline 2 & DHMS & $535.30 * *$ & $240.07 * *$ & $9.57 * *$ & $1.24 * *$ & $-12.31 * *$ & -0.04 & $4.99 * *$ & $3.55^{* *}$ & $2.47 * *$ & 1.77 & -0.11 & 1.03 & 0.35 \\
\hline 3 & RAH-13-86 & -6.60 & -6.04 & 1.14 & -0.18 & $8.81 * *$ & 0.15 & $3.78 * *$ & $3.11^{* * *}$ & 1.41 & 1.32 & 0.51 & 0.53 & 0.05 \\
\hline 4 & RAH-16 & $-303.36^{* *}$ & $-36.34 * *$ & $-5.67 * *$ & -0.45 & $-12.81 * *$ & 0.11 & $-4.86 * *$ & $-3.37 * *$ & 1.03 & -0.79 & -0.13 & 0.31 & 0.08 \\
\hline 5 & RAH-25-17 & $-138.41 * *$ & $-31.54 * *$ & 0.64 & -0.06 & $8.56 * *$ & 0.24 & 0.19 & 1.75 & $-1.81 * *$ & -1.36 & -0.20 & -0.60 & -0.07 \\
\hline 6 & RAH-370 & $72.31 * *$ & -9.64 & -0.64 & -0.43 & -0.94 & -0.27 & $-2.57 * *$ & $2.07 *$ & -0.70 & -0.29 & 0.26 & -0.43 & -0.39 \\
\hline 7 & RAH-5-10 & $-408.75^{* *}$ & $-145.28 * *$ & $-4.14 * *$ & -0.49 & $7.94 * *$ & 0.01 & -1.19 & $2.03 *$ & -0.57 & -0.46 & -0.31 & -0.28 & -0.09 \\
\hline \multirow[t]{5}{*}{8} & VB-37 & $-172.90 * *$ & $-95.97 * *$ & -1.53 & -0.17 & 2.94 & 0.01 & -0.49 & $-7.91 * *$ & -1.04 & 0.25 & -0.28 & -0.02 & 0.65 \\
\hline & SE(gi) & 125.32 & 44.78 & 1.36 & 0.12 & 2.15 & 0.10 & 0.75 & 0.85 & 0.43 & 0.85 & 0.25 & 0.27 & 0.44 \\
\hline & CD5\% & 24.34 & 14.55 & 2.54 & 0.77 & 3.19 & 0.68 & 1.88 & 2.01 & 1.43 & 2.01 & 1.10 & 1.13 & 1.44 \\
\hline & CD1\% & 29.62 & 17.70 & 3.08 & 0.93 & 3.88 & 0.83 & 2.29 & 2.44 & 1.74 & 2.44 & 1.33 & 1.37 & 1.75 \\
\hline & SEd (gi-gj) & 133.97 & 47.87 & 1.45 & 0.13 & 2.30 & 0.11 & 0.80 & 0.91 & 0.46 & 0.91 & 0.27 & 0.29 & 0.47 \\
\hline \multicolumn{15}{|c|}{ Barabadense testers } \\
\hline 1 & DB534 & $178.84 * *$ & $46.04 * *$ & $3.30 * *$ & 0.29 & $4.06 * *$ & -0.05 & $2.57 * *$ & $-2.01 * *$ & 0.51 & -0.39 & -0.35 & 0.16 & 0.01 \\
\hline 2 & SNICB75-10 & $46.46^{* *}$ & $34.38 * *$ & -0.79 & -0.04 & $3.63 * *$ & -0.15 & -0.30 & 1.10 & 0.29 & 0.20 & 0.04 & 0.23 & 0.30 \\
\hline 3 & RAB-4 & $-151.75 * *$ & $-63.34 * *$ & $-3.97 * *$ & -0.27 & -1.44 & 0.05 & -1.71 & -0.01 & -0.29 & -0.12 & 0.16 & -0.12 & -0.11 \\
\hline \multirow[t]{5}{*}{4} & RAB-8 & $-73.54 * *$ & $-17.09 *$ & 1.46 & 0.01 & $-6.25 * *$ & 0.16 & -0.56 & 0.92 & -0.51 & 0.31 & 0.16 & -0.28 & -0.19 \\
\hline & SE(gi) & 82.04 & 29.32 & 0.89 & 0.08 & 1.41 & 0.06 & 0.49 & 0.56 & 0.28 & 0.56 & 0.17 & 0.18 & 0.29 \\
\hline & CD5\% & 22.85 & 13.66 & 2.38 & 0.72 & 2.99 & 0.64 & 1.77 & 1.88 & 1.34 & 1.89 & 1.03 & 1.06 & 1.35 \\
\hline & CD1\% & 30.96 & 18.51 & 3.22 & 0.97 & 4.06 & 0.86 & 2.39 & 2.55 & 1.82 & 2.56 & 1.40 & 1.43 & 1.82 \\
\hline & SEd (gi-gj) & 189.47 & 67.70 & 2.05 & 0.19 & 3.25 & 0.15 & 1.13 & 1.29 & 0.65 & 1.29 & 0.39 & 0.41 & 0.66 \\
\hline
\end{tabular}


Table.3 Specific combining ability effects of crosses involving hirsutum and barbadense groups

\begin{tabular}{|c|c|c|c|c|c|c|c|c|c|c|c|c|c|c|}
\hline Sl no. & Crosses & $\begin{array}{c}\text { Seed } \\
\text { cotton } \\
\text { yield }(\mathrm{kg} \\
\text { /ha) }\end{array}$ & $\begin{array}{l}\text { Lint } \\
\text { yield (kg } \\
\text { /ha) }\end{array}$ & $\begin{array}{c}\text { Number } \\
\text { of bolls } \\
\text { per } \\
\text { plant }\end{array}$ & $\begin{array}{l}\text { Boll } \\
\text { weight } \\
\text { (g) }\end{array}$ & $\begin{array}{l}\text { Plant } \\
\text { height } \\
(\mathrm{cm})\end{array}$ & $\begin{array}{l}\text { Number of } \\
\text { monopodia }\end{array}$ & $\begin{array}{c}\text { Number } \\
\text { of } \\
\text { sympodia }\end{array}$ & $\begin{array}{c}\text { Sympodial } \\
\text { length at } 50 \\
\% \text { plant } \\
\text { height }(\mathrm{cm})\end{array}$ & $\begin{array}{c}\text { Ginning } \\
\text { outturn } \\
(\%)\end{array}$ & $\begin{array}{l}\text { Spadmeter } \\
\text { reading }\end{array}$ & $\begin{array}{c}\text { Inter } \\
\text { branch } \\
\text { distance } \\
(\mathrm{cm})\end{array}$ & $\begin{array}{l}\text { Lint } \\
\text { index } \\
(\mathrm{g})\end{array}$ & $\begin{array}{c}\text { Seed } \\
\text { index } \\
(\mathrm{g})\end{array}$ \\
\hline 1 & DH2752 x DB534 & $98.7 * *$ & $-53.2 * *$ & 1.21 & 0.59 & $-6.06 * *$ & 0.05 & 0.04 & $-8.37 * *$ & $-2.94 * *$ & 0.01 & -0.79 & -0.87 & -0.09 \\
\hline 2 & DH2752 x SNICB75-10 & $176.5 * *$ & $101.2 * *$ & $6.74 * *$ & 0.12 & $13.88^{* *}$ & -0.18 & 0.59 & $3.64 * *$ & 0.59 & $2.68 * *$ & -0.73 & -0.13 & -0.57 \\
\hline 3 & DH2752 x RAB4 & $-116.6 * *$ & $-104.7 * *$ & $-5.33 * *$ & -0.98 & $7.44 * *$ & 0.02 & 1.17 & $-4.09 * *$ & -0.06 & -1.13 & 0.46 & 0.21 & 0.54 \\
\hline 4 & DH2752 x RAB8 & $-158.6 * *$ & $56.6 * *$ & -2.61 & 0.28 & $-15.25 * *$ & 0.11 & -1.79 & $8.82 * *$ & $2.41 * *$ & -1.56 & 1.06 & 0.79 & 0.12 \\
\hline 5 & DHMS x DB534 & $-29.7 *$ & $18.3^{*}$ & 2.62 & -0.05 & $17.56^{* * *}$ & 0.55 & $-2.45^{*}$ & $3.61 * *$ & 0.41 & -2.05 & -0.16 & 0.85 & 1.29 \\
\hline 6 & DHMS x SNICB75-10 & $358.0 * *$ & $173.2 * *$ & $5.61 * *$ & 0.88 & $7.50 * *$ & $-1.27 * *$ & $5.42 * *$ & $9.17 * *$ & $4.23 * *$ & $6.91 * *$ & 0.75 & 1.15 & -0.50 \\
\hline 7 & DHMS x RAB-4 & $-376.7 * *$ & $-184.4 * *$ & $-6.51 * *$ & -0.20 & $6.56^{* * *}$ & 0.48 & $-2.66^{*}$ & $-7.14 * *$ & $-4.44 * *$ & $-5.42 * *$ & -1.27 & $-2.03 * *$ & -1.14 \\
\hline 8 & DHMS x RAB8 & $48.4 * *$ & -7.1 & -1.72 & -0.63 & $-31.63 * *$ & 0.24 & -0.31 & $-5.64 * *$ & -0.21 & 0.56 & 0.68 & 0.03 & 0.34 \\
\hline 9 & RAH-13-86 x DB534 & $100.0 * *$ & $-148.5 * *$ & $-5.15^{* *}$ & -0.63 & $-23.06^{* *}$ & -0.69 & -1.90 & $2.88 * *$ & $-2.11 * *$ & 0.62 & 0.91 & -1.04 & -0.71 \\
\hline 10 & RAH-13-86 x SNICB75-10 & $112.6 * *$ & $121.5 * *$ & 2.94 & 0.10 & -1.63 & 0.13 & $3.71 * *$ & $-8.31 * *$ & -0.87 & $-3.22 * *$ & -0.53 & -0.35 & 0.01 \\
\hline 11 & RAH-13-86 x RAB-4 & $247.1 * *$ & $197.9 * *$ & $7.82 * *$ & 0.58 & $18.94 * *$ & 0.16 & 2.55 & $3.80 * *$ & $6.61^{* *}$ & $7.37 * *$ & -1.04 & $2.30 * *$ & 0.21 \\
\hline 12 & RAH-13-86 x RAB8 & $-459.7 * *$ & $-170.9 * *$ & $-5.61 * *$ & -0.05 & $5.75^{* *}$ & 0.40 & $-4.35 * *$ & 1.63 & $-3.64 * *$ & $-4.77 * *$ & 0.66 & -0.92 & 0.49 \\
\hline 13 & RAH-16 x DB534 & $-577.5 * *$ & $-142.6 * *$ & $-3.45^{*}$ & -0.78 & $16.56^{* * *}$ & 0.45 & -1.84 & -1.39 & -0.99 & -1.54 & 0.75 & 0.09 & 0.76 \\
\hline 14 & RAH-16 x SNICB75-10 & 433.6** & $142.9 * *$ & -0.57 & 0.47 & $-39.00 * *$ & -0.07 & $-2.81 * *$ & $-3.50 * *$ & $2.04 * *$ & 0.19 & -0.04 & -0.05 & -1.27 \\
\hline 15 & RAH-16 x RAB-4 & $512.5 * *$ & 72.4** & $5.22 * *$ & 0.70 & -1.94 & -0.20 & 0.53 & $7.87 * *$ & -0.87 & 0.32 & 0.10 & 0.39 & 1.29 \\
\hline 16 & RAH-16 x RAB8 & $-368.7 * *$ & $-72.7 * *$ & -1.21 & -0.38 & $24.38 * *$ & -0.18 & $4.13 * *$ & $-2.98 * *$ & -0.18 & 1.03 & -0.81 & -0.43 & -0.78 \\
\hline 17 & RAH-25-17 x DB534 & $688.5 * *$ & $213.1^{* *}$ & $3.93 * *$ & $1.05^{*}$ & 1.19 & 0.20 & 1.52 & 0.74 & $1.89 *$ & -1.57 & -0.12 & 0.71 & 0.41 \\
\hline 18 & RAH-25-17 x SNICB75-10 & $-369.4 * *$ & $-142.9 * *$ & -3.10 & -0.49 & $7.63 * *$ & 0.21 & 1.34 & 0.28 & -1.70 & -1.53 & 0.48 & -0.77 & -0.62 \\
\hline 19 & RAH-25-17 x RAB4 & $-339.9 * *$ & -3.7 & $-4.98 * *$ & -0.32 & $-16.81 * *$ & -0.42 & 0.06 & $6.58 * *$ & -0.78 & 1.24 & 0.67 & -0.21 & 0.09 \\
\hline 20 & RAH-25-17 x RAB8 & 20.9 & $-66.5 * *$ & $4.14 * *$ & -0.24 & $8.00 * *$ & 0.02 & $-2.92 * *$ & -7.60 ** & 0.59 & 1.86 & -1.03 & 0.26 & 0.12 \\
\hline 21 & RAH-370 x DB534 & $-582.4 * *$ & $-104.2 * *$ & $-3.68 *$ & -0.58 & -0.81 & -0.07 & $3.11 * *$ & $5.01 * *$ & 1.05 & 1.52 & -0.09 & 0.29 & -0.02 \\
\hline 22 & RAH-370 x SNICB75-10 & $273.6^{* * *}$ & -5.6 & -2.69 & -0.14 & 0.63 & 0.08 & 1.14 & $-4.02 * *$ & -0.59 & -1.67 & -0.73 & -0.35 & -0.36 \\
\hline 23 & RAH-370 x RAB-4 & $432.3 * *$ & $169.0^{* * *}$ & 2.9 & 0.48 & $-12.81 * *$ & -0.42 & -0.72 & $-2.57 *$ & 0.02 & -1.08 & 0.51 & 0.15 & 0.35 \\
\hline 24 & RAH-370 x RAB-8 & $-123.5 * *$ & $-59.1 * *$ & $3.37 *$ & 0.25 & $13.00 * *$ & 0.42 & $-3.54 * *$ & 1.59 & -0.48 & 1.23 & 0.31 & -0.09 & $\mathbf{0 . 0 3}$ \\
\hline 25 & RAH-5-10 x DB534 & $190.8^{* *}$ & $100.9^{* * *}$ & 1.08 & 0.10 & -0.69 & -0.15 & -0.76 & -1.20 & 1.71 & 2.13 & -0.46 & 0.21 & -0.57 \\
\hline 26 & RAH-5-10 x SNICB75-10 & $-419.1 * *$ & $-161.3 * *$ & $-4.29 * *$ & -0.23 & 3.25 & 0.43 & $-3.30 * *$ & $6.69^{* *}$ & $-2.27 * *$ & $-3.06 * *$ & 0.75 & 0.11 & 1.64 \\
\hline 27 & RAH-5-10 x RAB4 & $-216.4 * *$ & $-50.2 * *$ & 1.20 & -0.35 & -1.69 & 0.28 & -0.89 & $-3.37 * *$ & -0.36 & -0.96 & 0.33 & -0.44 & -0.70 \\
\hline 28 & RAH-5-10 x RAB-8 & $444.6^{* * *}$ & $110.6^{* *}$ & 2.02 & 0.48 & -0.88 & -0.56 & $4.96 * *$ & -2.12 & 0.93 & 1.88 & -0.62 & 0.12 & -0.37 \\
\hline 29 & VB-37 x DB534 & $111.6^{* * *}$ & $116.1 * *$ & $3.45^{*}$ & 0.31 & $-4.69 * *$ & -0.35 & 2.29 & -1.27 & 0.98 & 0.87 & -0.05 & -0.25 & -1.06 \\
\hline 30 & VB-37 x SNICB75-10 & $-565.8 * *$ & $-228.9 * *$ & $-4.65 * *$ & -0.70 & $7.75^{* * *}$ & 0.68 & $-6.10 * *$ & $-3.96 * *$ & -1.42 & -0.30 & 0.06 & 0.39 & 1.66 \\
\hline 31 & VB-37 x RAB4 & $-142.4 * *$ & $-96.2 * *$ & -0.41 & 0.09 & 0.31 & 0.10 & -0.03 & -1.09 & -0.13 & -0.33 & 0.25 & -0.37 & -0.64 \\
\hline \multirow[t]{5}{*}{32} & VB-37 x RAB-8 & $596.6^{* *}$ & $208.9^{* * *}$ & 1.61 & 0.29 & -3.38 & -0.43 & $3.84 * *$ & $6.31 * *$ & 0.58 & -0.24 & -0.26 & 0.23 & 0.04 \\
\hline & SE (gi) & 217.06 & 77.56 & 2.35 & 0.22 & 3.73 & 0.17 & 1.30 & 1.47 & 0.75 & 1.48 & 0.44 & 0.47 & 0.76 \\
\hline & CD 5\% & 29.76 & 17.79 & 3.10 & 0.94 & 3.90 & 0.83 & 2.30 & 2.45 & 1.75 & 2.46 & 1.34 & 1.38 & 1.75 \\
\hline & CD 1\% & 34.51 & 20.63 & 3.59 & 1.09 & 4.52 & 0.97 & 2.67 & 2.84 & 2.03 & 2.85 & 1.56 & 1.60 & 2.04 \\
\hline & SEd (gi-gj) & 401.92 & 143.62 & 4.36 & 0.40 & 6.90 & 0.32 & 2.40 & 2.73 & 1.38 & 2.74 & 0.82 & 0.86 & 1.40 \\
\hline
\end{tabular}


The hirsutum line DHMS (2.47) showed significant positive gca effect for ginning outturn. Whereas, lines RAH-25-17 (-1.87) exhibited significant negative gca effects. None of the testers showed significant positive or negative gca effects. Among the crosses positive sca effects ranged from 1.89 (RAH-5-10 x DB534) to 6.61 (RAH-13-86 x RAB-4) significant negative sca effects ranged from -2.11 (RAH-13-86 x DB534) to 4.44 (DHMS x RAB-4). The crosses viz., RAH-13-86 x RAB4 (6.61), DHMS $x$ SNICB75-10 (4.23) and DHMS $x$ RAB8 (2.41) exhibited highest significant positive sca effects. The above results were in accordance with Neelima (2002), Maisuria et al. (2006) and Patil (2009) for this trait.

None of the lines or testers had significant gca effects for SPAD meter reading. Among crosses, sca effects were in the range of -5.42 (VB-37 x SNICB75-10) to 7.37 (DHMS $x$ RAB-4) and three crosses viz., DHMS $x$ RAB-4 (7.37), DHMS x SNICB75-10 (6.91) and DH2752 $x$ SNICB75-10 (2.68) had significant positive sca effects for this trait. None of the lines or testers had significant gca effects for this trait for inter branch distance, lint index and seed index. The range of sca effects for inter branch distance varied from 1.27 (DHMS x RAB-4) to 1.06 (DH2752 x RAB8) and no cross had significant sca effects for this trait. For lint index sca effects were in the range of -2.03 (DHMS x RAB8) to 2.30 (RAH-13-86 x RAB-4) and only two crosses viz., DHMS x RAB8 (-2.03) and RAH-13-86 x RAB-4 (2.30) had significant sca effects. For seed index sca effects for crosses were in the range of -1.14 (DHMS $x$ RAB-4) to 1.66 (VB-37 x DB534) and no cross had significant sca effects for this trait.

The estimates of variances in combining ability analysis of the present study revealed the preponderance of both non-additive and additive $\mathrm{x}$ non-additive gene action for most of the characters which can be exploited by development of interspecific hybrids between the opposite heterotic groups. The results of combining ability effects revealed that the parent DHMS exhibited significant gca effects for most of the characters indicating the usefulness of this parent as best hirsutum line in developing the interspecific hybrids. Similarly, SNICB75-10 is the best barbadense parent to be used as tester. The combining ability status of most productive crosses in this study helped in drawing the inference about handling the best crosses identified in the present study. Results of specific combining ability effects indicated that the crossesDHMS x SNICB75-10 andDH-2752 x SNICB75-10 were best specific combiners in the present study. This also indicated that best general combiners have given best specific combination for seed cotton yield.Based on their per se performance and combining ability effectsthese hybrids can be used to derive superior segregants infurther generations and deriving the hybrid oriented populations in cotton.

\section{Acknowledgement}

The authors are great full to the Technology Mission on Cotton, Mini Mission-I initiated with financial outlay from Department of Agriculture and Cooperation, Ministry of Agriculture, Government of India and implemented by the UAS, Dharwad for financial support.

\section{References}

Anonymous, 2017. Status Paper of Indian Cotton, Directorate of Cotton Development Government of India Ministry of Agriculture \& Farmers Welfare, DAC \& FW, Nagpur 440013, Maharashtra.

Kajjidoni, S.T., 1997. Histological basis of genetic male sterility and its utilization in hybrid development in diploid cottons. $\mathrm{Ph}$. D. Thesis, Univ. Agric. Sci., Dharwad, 
Karnataka.

Kaushik and Kapoor, C.J., (2007) Genetics of yield and other traits over environments in American cotton (Gossypium hirsutum L.). J. Cotton Res. Dev., 21: 6-11.

Kempthorne, O. 1957.An introduction to genetic statistics, New York, John Willey and Sons Inc. London, Chapman and Hall Ltd.

Lavanyakumar, K. R., 2004, Genetic studies on plant types of cotton (Gossypium hirsutum L.) M. Sc. (Agri.) Thesis, Univ. Agric. Sci., Dharwad, Karnataka (India).

Maisuria, A.T., Patel, J.C., Patel, K.G. and Solanki, B.G., (2006) Study of best per se performance, heterosis and combining ability effects for seed cotton yield and its component characters through GMS system in Asiatic cotton. J. Indian Soc. Cotton Improv., 3: 88-91.

Mallikarjun, H.B., 2005. Evaluation of recombinational variability for combining ability in cotton. Ph. D Thesis, Univ. Agric. Sci., Dharwad, Karnataka.

Neelima, S., 2002.Heterosis and combining ability analysis for yield and yield components in cotton (Gossypium hirsutum L.). M. Sc. (Agri.) Thesis, Acharya N.G. Ranga Agric. Univ. Rajendranagar, Hyderabad (India).

Nidagundi, J. M., 2010, Genetic studies on productivity and quality features in cotton (G.hirsutum L.) Ph. D. Thesis, Univ. Agric. Sci, Dharwad, Karnataka (India).

Patil, S. S., 2009. Bt. Cotton: opportunities and prospects. Proceedings of National symposium, CICR, Nagpur, November 1719.

Pole, S. P., 2008. Sudewad, S. M., Kamble, S. K. and Borgaonkar, S. B.: Heterosis for seed cotton yield and yield components in upland cotton (Gossypium hirsutum L.). J. Cotton Res. Dev., 22: 139-142.
Pranesh, K.J., 2014. Exploiting heterotic groups through reciprocal recurrent selection for combing ability to improve performance of cotton hybrids, Ph. D. Thesis, Univ. Agric. Sci, Dharwad, Karnataka (2014).

Rajeev.S., 2011.Relating phenotyping and molecular diversity with heterosis in cotton (Gossypium hirsutum). M. Sc (Agri.) Thesis, Univ. Agric. Sci., Dharwad, Karnataka (India).

Rama Khrishna, 2008, Reciprocal selection for combining ability in cotton $\mathrm{Ph}$. D. Thesis, Univ. Agric. Sci, Dharwad, Karnataka.

Reddy, A.N. 2001.Heterosis, combining ability and stability analysis of hybrids for yield and yield components in cotton (Gossypium hirsutum L.). Ph. D. Thesis, Acharya N. G. Ranga Agric. Univ., Hyderabad.

Saifullah, A. et al., 2014. To estimates heterosis and heterobeltosis of yield and quality traits in upland cotton. J. Bio. Agril.and Healthcare., 4: 2224 -2231.

Shanmugavalli, N. and Vijendradas, L.D. 1995, Line $\mathrm{x}$ tester analysis of combining ability in cotton. Madras Agric. J., 82: 449-452.

Somashekhar, D. 2006, Genetic studies on reciprocal selection for combining ability to improve hybrid performance in cotton. $\mathrm{Ph}$. D Thesis, Univ. Agric. Sci., Dharwad, Karnataka.

Sprague, G.F. and Tatum, L.A., 1942. General and specific combining ability in single crosses in corn. J. American Soc. Agron., 34: 923-932.

Yanal, A. A., 2013, Development and exploitation of Heterotic pools of G.hirsutum and G.barbadense for developing potential interspecific hybrids, molecular marker and transformation study in cotton. Ph.D. Thesis, Univ. Agric. Sci, Dharwad, Karnataka.

\section{How to cite this article:}

Rajeev, S. and Patil, S.S. 2018. Combining Ability Studies in Cotton Interspecific Heterotic Group Hybrids ( $G$. hirsutum x $G$. barbadense) for Seed Cotton Yield and its Components. Int.J.Curr.Microbiol.App.Sci. 7(07): 3955-3963. doi: https://doi.org/10.20546/ijcmas.2018.707.460 